This is an electronic reprint of the original article. This reprint may differ from the original in pagination and typographic detail.

Author(s): Tarpanov, D.; Dobaczewski, Jacek; Toivanen, Jussi; Carlsson, B. G.

Title: $\quad$ Spectroscopic Properties of Nuclear Skyrme Energy Density Functionals

Year: $\quad 2014$

Version:

Please cite the original version:

Tarpanov, D., Dobaczewski, J., Toivanen, J., \& Carlsson, B. G. (2014). Spectroscopic Properties of Nuclear Skyrme Energy Density Functionals. Physical review letters, 113(25), Article 252501. https://doi.org/10.1103/PhysRevLett.113.252501

All material supplied via JYX is protected by copyright and other intellectual property rights, and duplication or sale of all or part of any of the repository collections is not permitted, except that material may be duplicated by you for your research use or educational purposes in electronic or print form. You must obtain permission for any other use. Electronic or print copies may not be offered, whether for sale or otherwise to anyone who is not an authorised user. 


\title{
Spectroscopic Properties of Nuclear Skyrme Energy Density Functionals
}

\author{
D. Tarpanov, ${ }^{1,2}$ J. Dobaczewski, ${ }^{1,3,4}$ J. Toivanen, ${ }^{3}$ and B. G. Carlsson ${ }^{5}$ \\ ${ }^{1}$ Institute of Theoretical Physics, Faculty of Physics, University of Warsaw, Pasteura 5, PL-02-093 Warsaw, Poland \\ ${ }^{2}$ Institute for Nuclear Research and Nuclear Energy, 1784 Sofia, Bulgaria \\ ${ }^{3}$ Department of Physics, University of Jyväskylä, P.O. Box 35 (YFL), FI-40014 Jyväskylä, Finland \\ ${ }^{4}$ Helsinki Institute of Physics, University of Helsinki, P.O. Box 64, FI-00014 Helsinki, Finland \\ ${ }^{5}$ Division of Mathematical Physics, LTH, Lund University, P.O. Box 118, S-22100 Lund, Sweden
}

(Received 19 May 2014; published 16 December 2014)

\begin{abstract}
We address the question of how to improve the agreement between theoretical nuclear single-particle energies (SPEs) and observations. Empirically, in doubly magic nuclei, the SPEs can be deduced from spectroscopic properties of odd nuclei that have one more or one less neutron or proton. Theoretically, bare SPEs, before being confronted with observations, must be corrected for the effects of the particle vibration coupling (PVC). In the present work, we determine the PVC corrections in a fully self-consistent way. Then, we adjust the SPEs, with PVC corrections included, to empirical data. In this way, the agreement with observations, on average, improves; nevertheless, large discrepancies still remain. We conclude that the main source of disagreement is still in the underlying mean fields, and not in including or neglecting the PVC corrections.
\end{abstract}

DOI: 10.1103/PhysRevLett.113.252501

PACS numbers: 21.10.Pc, 21.60.Jz

Finite many-fermion systems, such as quantum dots, ultracold Fermi gases, atoms, or atomic nuclei, exhibit conspicuous shell effects. These can easily be modeled within mean-field approaches, which assume that fermions occupy single-particle states in a common one-body potential. In nuclei, the ensuing shell effects are responsible not only for sequences of excited states in odd nuclei, and for their quadrupole or magnetic moments, but also for deformation properties, including the fission phenomena, or detailed features of rotational bands [1-4].

A precise description of nuclear spectroscopic properties, that is, those pertaining to single-particle structures, is one of the most important goals of theory. The theoretical approach that is particularly well suited to describe these structures is the energy-density-functional (EDF) formalism, wherein the Kohn-Sham single-particle orbitals play an essential role. Although the Kohn-Sham single-particle energies (SPEs), or bare SPEs, have, in principle, only an auxiliary meaning, see, e.g., the recent analysis in Ref. [5], in nuclei they do provide a fair description of masses and excited states of odd nuclei. The question of quantitative determination of many-body corrections to nuclear SPEs is a matter of ongoing debate, see Refs. [6-11].

In practice, all nuclear EDFs currently used in applications depend on parameters or coupling constants adjusted to empirical data $[12,13]$. In addition, most of them were constructed by adjusting bare SPEs to selected empirical information. Therefore, it is not at all clear to what extent the many-body corrections were, or were not, included in the EDFs' parameters, and thus whether it is legitimate to add them $a$ posteriori.
In the present Letter, we take up the challenge of adjusting EDFs' parameters to empirical SPEs after having added many-body corrections. This is certainly the right way of proceeding, which was never tried up to now, and which allows us to study the interplay between the meanfield and beyond-mean-field effects on the SPEs.

We determined the many-body corrections to SPEs within the standard particle-vibration-coupling (PVC) model [1,6,14-16], which is based on coupling particles and holes with the random-phase-approximation (RPA) phonons up to second order of perturbation theory. The calculations were performed in a fully self-consistent way, that is, the same Skyrme EDF parametrization was used to determine the ground states of even-even nuclei, singleparticle states, RPA phonons, and particle-phonon vertices. The PVC correction $\delta \epsilon_{i}$ to the SPE $\epsilon_{i}$ of the $i$ th state has the form [6]

$$
\begin{aligned}
\delta \epsilon_{i}= & \frac{1}{2 j_{i}+1}\left(\sum_{n J p} \frac{|\langle i\|V\| p, n J\rangle|^{2}}{\epsilon_{i}-\epsilon_{p}-\hbar \omega_{n J}+i \eta}\right. \\
& \left.+\sum_{n J h} \frac{|\langle i\|V\| h, n J\rangle|^{2}}{\epsilon_{i}-\epsilon_{h}+\hbar \omega_{n J}-i \eta}\right),
\end{aligned}
$$

where $\langle i\|V\| p, n J\rangle$ and $\langle i\|V\| h, n J\rangle$ are, respectively, the standard particle-phonon and hole-phonon vertex reduced matrix elements. Similarly as in Ref. [6], a small imaginary parameter is added to the denominator with $\eta=0.05 \mathrm{MeV}$.

Let us briefly discuss the physical contents of the PVC correction (1). A rigorous density functional theory formalism based on the Hohenberg-Kohn [17] and KohnSham [18] theorems stipulates that there exists an exact 
universal functional, which should give the exact lowest energies (in each quantum number) of even and odd nuclei, which can be directly compared with experimental masses. Needless to say, such an exact functional is not known. However, we know that when a phenomenological EDF is minimized in even and odd nuclei, the resulting odd-even mass differences are not equal to the Kohn-Sham energies, see the recent Ref. [19] for discussions and further references. Then, the so-called polarization corrections to particle and hole SPEs are equal to diagonal terms in Eq. (1), for $i=p$ and $i=h$, respectively. Full PVC correction (1) can thus be regarded as an approximate way to generalize our functional so as to model the degrees of freedom associated with the mixing of the odd particle with particle-vibration coupled states.

In the present Letter, we concentrate on presenting results obtained for the bare and PVC-corrected SPEs. As discussed above, the former ones do not have physical meaning; however, they provide us with a simple illustration of one-body nuclear properties, and we show them below as an important background that facilitates communication and comparison of results.

As a baseline of our analysis, we used a set of five different Skyrme EDF parametrizations, SAMi [20], SLy5 [21], SIII [22], SkM* [23], and SkP [24], which are characterized by quite different effective masses, ranging from $m^{*} / m=0.675$ to 1 . We carried out the calculations using the spherical solver HOSPHE $[25,26]$, in which the determination of the PVC corrections was implemented [27]. The mean-field, RPA, and PVC solutions were obtained with a harmonic-oscillator basis using 15 oscillator shells (17 shells for ${ }^{208} \mathrm{~Pb}$ ).

We included effects of phonons with both parities and considered multipolarities ranging from $J=0$ to 15 , although only for phonons up to $J=6$ we obtained a significant impact on the results. The PVC corrections were determined in the single-particle and phonon spaces restricted to below 15 and $30 \mathrm{MeV}$, respectively. In addition, only significantly collective phonons, that is, those contributing more than $5 \%$ to the non-energy-weighted sum rule of the given channel, were taken into account $[6,14]$. A detailed analysis of numerical conditions and convergence will be presented in the forthcoming publication [27].

In Figs. 1 and 2, we show values of the PVC corrections calculated for neutron and proton SPEs, respectively, in six doubly magic nuclei ${ }^{16} \mathrm{O},{ }^{40} \mathrm{Ca},{ }^{48} \mathrm{Ca},{ }^{56} \mathrm{Ni},{ }^{132} \mathrm{Sn}$, and ${ }^{208} \mathrm{~Pb}$. Those values are also tabulated in the Supplemental Material [28]. We see that in some cases (e.g., $j=1 / 2$ states in ${ }^{56} \mathrm{Ni}$ ), the largest (smallest) PVC corrections are obtained for the smallest (largest) effective masses; however, the pattern of PVC corrections depends strikingly weakly on the EDF parametrization.

Experimentally, the SPEs are not measurable quantities and they cannot be defined in an entirely model-independent way [5]. They are usually associated

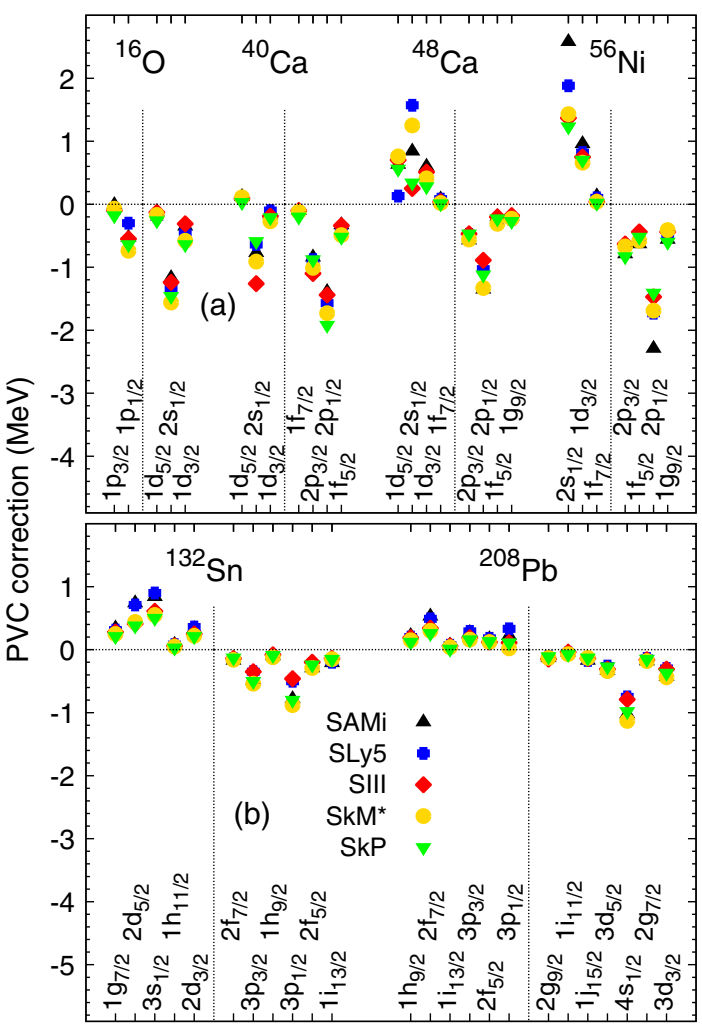

FIG. 1 (color online). PVC corrections calculated for neutron SPEs in six doubly magic nuclei and for five parametrizations of the Skyrme EDF. For each nucleus, thin vertical lines separate hole and particle states. (a) and (b) show results for light and heavy nuclei, respectively.

with masses and spectra of odd nuclei by considering the so-called spectroscopic factors related to probabilities of one-nucleon transfer reactions. Different analyses of this type exist in the literature, and for the purpose of the present study, we use those of Grawe et al. [31-33] (data set $A$ ), Schwierz et al. [34] (data set B), and Porquet et al. [35-37] (data set $C$ ). In addition, we also compare our results to two derived or reduced data sets: (i) data set $M$, which contains average values of SPEs simultaneously listed in data sets $A, B$, and $C$, provided the three energies agree with the average values within $200 \mathrm{keV}$, and (ii) data set $S$, which contains a subset of data set $B$ for spectroscopic factors larger than 0.8. In this way, data set $M$ contains SPEs, for which the three evaluations agree best, and data set $S$ contains those which correspond to least fragmented states. All data sets used in the present Letter are listed in the Supplemental Material [28].

In Fig. 3, we show residuals of bare and PVC-corrected SPEs, calculated with respect to empirical values of data set $A$. We see that both bare and PVC-corrected SPEs poorly agree with data, with deviations reaching up to around $4 \mathrm{MeV}$. The distributions of residuals are manifestly nonstatistical; hence, strong systematic effects are still present [38]. Clearly, PVC corrections do not improve the picture 


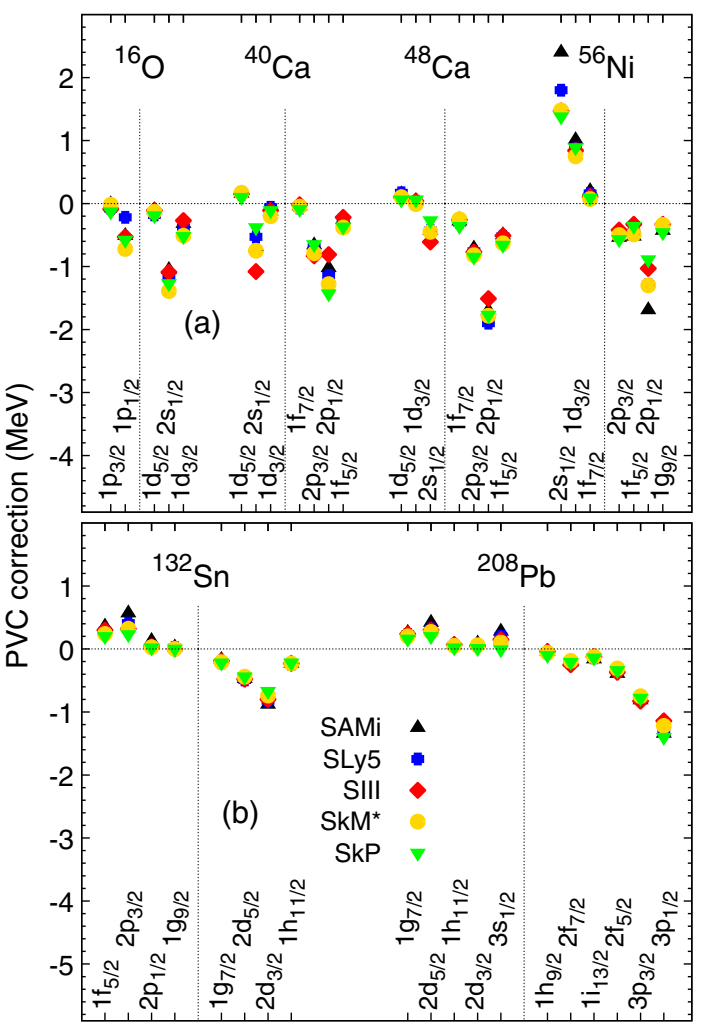

FIG. 2 (color online). Same as in Fig. 1 but for proton SPEs.

significantly. Simply adding the PVC corrections to bare SPEs calculated for standard EDFs is insufficient, and the readjustment of EDFs, as proposed in the present study, is mandatory.

To perform adjustments of the EDF coupling constants to empirical SPEs, we follow the methodology of

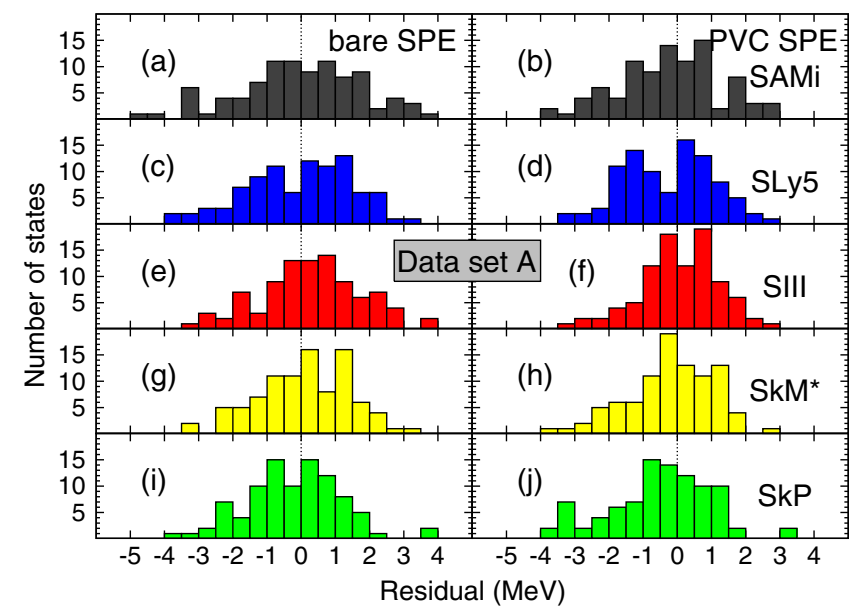

FIG. 3 (color online). Distributions of residuals of the bare (left panels) and PVC-corrected (right panels) SPEs, determined with respect to the empirical values of data set $A$, and shown for the five parametrizations of the Skyrme EDF: SAMi (a) and (b), SLy5 (c) and (d), SIII (e) and (f), SkM* (g) and (h), and SkP (i) and $(\mathrm{j})$. regression analysis, as it was applied in Ref. [39]. The method is based on the observation that the standard Skyrme energy density, see, e.g., Refs. [40,41], depends linearly on the 12 EDF coupling constants, $C_{m}$, $m=1, \ldots, 12$. In Ref. [39], it has been shown numerically that the above linear dependence carries over to an approximate linear dependence of bare SPEs on $C_{m}$. We are using this fact in order to build the regression matrix $I_{i m}=\partial \epsilon_{i} / \partial C_{m}$, where the partial derivatives are calculated using the finite-difference formula for SPEs $\epsilon_{i}$ corresponding to coupling constants $C_{m}^{0} \pm d_{m}$, perturbed by suitably small shifts $d_{m}$. In this way, we determine the regression matrices for coupling constants $C_{m}^{0}$ corresponding to five Skyrme EDF parametrizations considered in this study.

Using the regression matrices, and assuming that for reasonably small changes of the coupling constants they do not significantly change, one can fit the EDF coupling constants to the empirical SPEs. To this end, one must solve the set of linear equations, $r_{i}^{0}=\sum_{m} I_{i m} \Delta C_{m}$, where $r_{i}^{0}=$ $\epsilon_{i}^{0}-\epsilon_{i}^{\exp }$ are residuals of SPEs calculated for a given Skyrme EDF and $\Delta C_{m}$ are corrections to coupling constants. Since the numbers of empirical SPEs $(M=93,83$, 78,48 , and 49 for data sets $A, B, C, M$, and $S$, respectively) are larger than the number of coupling constants (12), the best approximation is obtained within the standard leastsquares method, see, e.g., Ref. [42], which minimizes the rms deviation between the theory and experiment, $\Delta \epsilon_{\text {rms }}=\left[(1 / M) \sum_{i=1}^{M}\left(\epsilon_{i}-\epsilon_{i}^{\exp }\right)^{2}\right]^{1 / 2}$.

Figures 4 and 5 summarize results obtained after the fits and compare them to those determined before the fits, that is, to those corresponding to the original five Skyrme EDF parametrizations. We note that the fitted values of SPEs were obtained directly from the regression analysis. Figure 4 shows partial rms deviations corresponding to

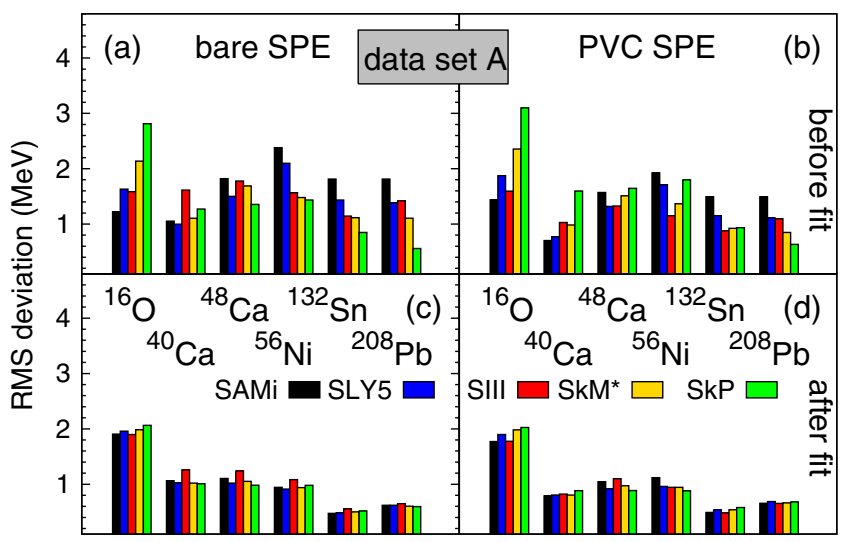

FIG. 4 (color online). Left: (a) and (c) [right (b) and (d)] panels: rms deviations between the bare [PVC-corrected] SPEs and empirical data of set A. Upper: (a) and (b) and lower: (c) and (d) panels show results obtained for standard Skyrme EDFs and for refitted parametrizations, respectively. In all cases, partial contributions obtained in six doubly magic nuclei are shown. 


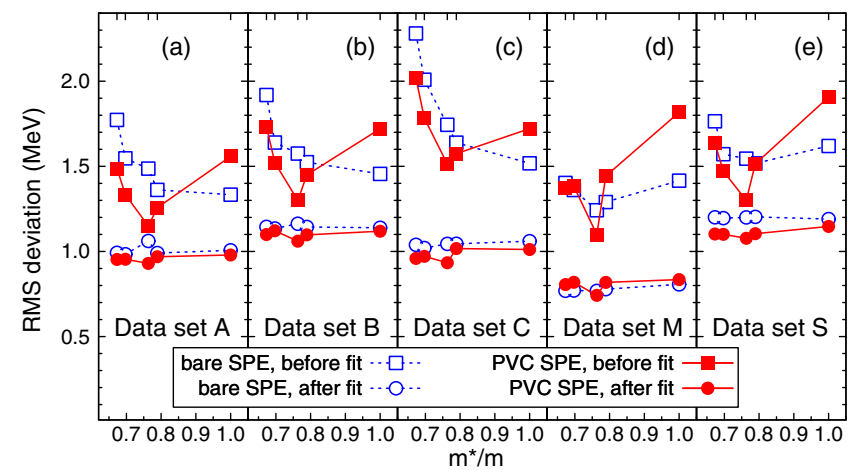

FIG. 5 (color online). Open (full) symbols: rms deviations between the bare (PVC-corrected) SPEs and empirical data of sets $A$ (a), $B$ (b), $C$ (c), $M$ (d), and $S$ (e) (see text). Results obtained for the five studied Skyrme EDFs (squares), and for the corresponding refitted parametrizations (circles), are shown in the function of the effective mass corresponding to the original Skyrme EDF parametrizations.

the six studied nuclei, whereas total rms deviations are shown in Fig. 5.

First, in Fig. 5 we see that the bare SPEs obtained for standard Skyrme EDFs exhibit a conspicuous effectivemass dependence, with those corresponding to $\mathrm{m}^{*} / \mathrm{m}=1$ being, on average, closest to data. This reflects the general feature of adjusting EDFs to ground-state properties, whereupon one systematically obtains the best fits for $m^{*} / m=1$ [13]. In Fig. 4(a) we also see that this trend is reversed in ${ }^{16} \mathrm{O}$ and absent in ${ }^{40,48} \mathrm{Ca}$, so the effect is clearly marked only in heavy nuclei.

Second, in Fig. 5 we see that for the $m^{*} / m \leq 1 \mathrm{EDFs}$, the PVC-corrected SPEs agree (before the fit) slightly better with empirical SPEs than the bare SPEs. The degree of improvement is systematic, but small-so small that in Fig. 3 it is not even really visible. For the $m^{*} / m=1$ EDF, where already the bare SPEs agree with data best, the PVC corrections lead to a deteriorated agreement.

Third, fits to bare and PVC-corrected SPEs, Figs. 4(c), 4(d), and 5, give rms deviations that are very weakly dependent on variants of the Skyrme EDF. This indeed confirms the validity of our regression analysis, because independently of the starting point, linearity of the problem allows for bringing all optimized SPEs to one common point. Note that these results indicate that the PVC corrections alone also depend on the coupling constants approximately linearly. The remaining weak dependence on the starting point may reflect possible small nonlinearities as well as the fact the studied EDFs are defined with different powers of the density dependence, which were not included in the regression analysis. At the same time, we should bear in mind that the regression analysis can be ill conditioned, cf. Ref. [43], that is, while giving robust values of the rms deviations, which are discussed in this Letter, it may give poorly defined values of model parameters.
Most important, in Fig. 5 we see that fits of neither bare nor PVC-corrected SPEs can bring us below the glass floor of about $1 \mathrm{MeV}$ of the total rms deviation. The independence of this limit to the PVC corrections being included or not, shows that they are not really giving us, on average, any better agreement with empirical SPE's. Moreover, it also shows that the impact of the PVC corrections on SPEs can be fairly well absorbed in the current parametrization of the Skyrme EDF.

As seen in Fig. 5, details of the comparison with observations are still dependent on the way the empirical SPEs are extracted from data. However, independently of which of the data sets $A, B$, or $C$ is used, the optimized results again do not go below the limit of about $1 \mathrm{MeV}$ rms deviation. In fact, the exact value of this limit depends on the selection of the empirical data [28] — considering only those data points where data sets $A, B$, and $C$ agree within $\pm 200 \mathrm{keV}$ (data set $M$ ), the rms goes down to about $0.8 \mathrm{MeV}$, and for those corresponding to spectroscopic factors larger than 0.8 (data set $S$ ), it stays at about $1.1 \mathrm{MeV}$, where it also was for data set $B$.

In conclusion, the findings of the present Letter cast some doubts on whether a rather poor agreement of bare SPEs, obtained within EDF approaches, can be improved by taking into account PVC corrections. We do not find any rationale in favor of adding these corrections on top of results obtained for functionals that were adjusted to observations without these corrections. In our study, we obtained results for functionals refitted after adding the PVC corrections. Although this was done within the approximation based on linear regression, one could not improve the results below the lower limit of about $1 \mathrm{MeV}$ rms deviation.

When fitting the coupling constants, we did not consider any other observables, such as nuclear masses or radii. Therefore, the obtained results simply illustrate the maximum possible improvement that can potentially be obtained in the description of the empirical SPEs. Taking into account other observables can only worsen the results obtained for SPEs, which makes the conclusions of our Letter even stronger. Certainly, PVC effects are needed for a correct description of fragmented single-particle strengths [44]; however, for a detailed description of values of SPEs, they do not lead to any dramatic improvement.

In our opinion, the burden of improving the current limited level of agreement of nuclear spectroscopic properties with data is still on the definition and form of the used EDFs [13], and not on higher-order perturbative corrections. Indeed, one cannot reasonably expect that manybody corrections can compensate for the rather rudimentary forms of EDFs currently in use. The search for better EDFs, which is currently pursued in various directions, remains an important priority for the field.

Private communications from H. Grawe, J. Dudek, and M.-G. Porquet, related to the empirical values of SPEs, are 
gratefully acknowledged. We thank Gianluca Colò and Witek Nazarewicz for critical reading of the manuscript and comments. This work was supported in part by the THEXO JRA within the EU-FP7-IA Project ENSAR (Grant No. 262010), by the Academy of Finland and University of Jyväskylä within the FIDIPRO program, by the Polish National Science Center under Contract No. 2012/07/B/ ST2/03907, by the Bulgarian Science Fund under Contract No. NuPNET-SARFEN DNS7RP01/0003, and by the ERANET-NuPNET grant SARFEN of the Polish National Centre for Research and Development (NCBiR). We acknowledge the CSC-IT Center for Science Ltd., Finland, for the allocation of computational resources. B. G. C. thanks the Swedish Research Council (VR) for financial support.

[1] A. Bohr and B. R. Mottelson, Nuclear Structure (W. A. Benjamin, New York, 1969), Vol. I.

[2] A. Bohr and B. R. Mottelson, Nuclear Structure (W. A. Benjamin, Reading, 1975), Vol. II.

[3] M. Brack, J. Damgaard, A. S. Jensen, H. C. Pauli, V. M. Strutinsky, and C. Y. Wong, Rev. Mod. Phys. 44, 320 (1972).

[4] Z. Szymański, Fast Nuclear Rotation (Clarendon Press, Oxford, 1983).

[5] T. Duguet and G. Hagen, Phys. Rev. C 85, 034330 (2012).

[6] G. Colò, H. Sagawa, and P. F. Bortignon, Phys. Rev. C 82, 064307 (2010).

[7] P. F. Bortignon, G. Colò, and H. Sagawa, J. Phys. G37, 064013 (2010).

[8] E. V. Litvinova and A. V. Afanasjev, Phys. Rev. C 84, 014305 (2011).

[9] A. Idini, F. Barranco, and E. Vigezzi, Phys. Rev. C 85, 014331 (2012).

[10] L.-G. Cao, G. Colò, H. Sagawa, and P. F. Bortignon, Phys. Rev. C 89, 044314 (2014).

[11] N. V. Gnezdilov, I. N. Borzov, E. E. Saperstein, and S. V. Tolokonnikov, Phys. Rev. C 89, 034304 (2014).

[12] M. Bender, P.-H. Heenen, and P.-G. Reinhard, Rev. Mod. Phys. 75, 121 (2003).

[13] M. Kortelainen, J. McDonnell, W. Nazarewicz, E. Olsen, P.-G. Reinhard, J. Sarich, N. Schunck, S. M. Wild, D. Davesne, J. Erler et al., Phys. Rev. C 89, 054314 (2014).

[14] V. Bernard and N. Van Giai, Nucl. Phys. A348, 75 (1980).

[15] C. Mahaux, P. F. Bortignon, R. A. Broglia, and C. H. Dasso, Phys. Rep. 120, 1 (1985).

[16] V. Van der Sluys, D. Van Neck, M. Waroquier, and J. Ryckebusch, Nucl. Phys. A551, 210 (1993).

[17] P. Hohenberg and W. Kohn, Phys. Rev. 136, B864 (1964).

[18] W. Kohn and L. J. Sham, Phys. Rev. 140, A1133 (1965).
[19] D. Tarpanov, J. Toivanen, J. Dobaczewski, and B. G. Carlsson, Phys. Rev. C 89, 014307 (2014).

[20] X. Roca-Maza, G. Colò, and H. Sagawa, Phys. Rev. C 86, 031306 (2012).

[21] E. Chabanat, P. Bonche, P. Haensel, J. Meyer, and R. Schaeffer, Nucl. Phys. A635, 231 (1998).

[22] M. Beiner, H. Flocard, N. Van Giai, and P. Quentin, Nucl. Phys. A238, 29 (1975).

[23] J. Bartel, P. Quentin, M. Brack, C. Guet, and H.-B. Håkansson, Nucl. Phys. A386, 79 (1982).

[24] J. Dobaczewski, H. Flocard, and J. Treiner, Nucl. Phys. A422, 103 (1984).

[25] B. G. Carlsson, J. Dobaczewski, J. Toivanen, and P. Veselý, Comput. Phys. Commun. 181, 1641 (2010).

[26] B. G. Carlsson, J. Toivanen, J. Dobaczewski, P. Veselý, Y. Gao, and D. Ward (to be published).

[27] D. Tarpanov et al. (to be published).

[28] See Supplemental Material at http://link.aps.org/ supplemental/10.1103/PhysRevLett.113.252501, which includes Refs. [29,30], for results pertaining to data sets $A, B$, $C, M$, and $S$, and for a comparison with experimental levels in odd nuclei.

[29] B. A. Brown, Phys. Rev. C 58, 220 (1998).

[30] Evaluated Nuclear Structure Data File, http://www.nndc.bnl .gov/ensdf/.

[31] H. Grawe, K. Langanke, and G. Martìnez-Pinedo, Rep. Prog. Phys. 70, 1525 (2007).

[32] T. Faestermann, M. Górska, and H. Grawe, Prog. Part. Nucl. Phys. 69, 85 (2013).

[33] H. Grawe (private communication).

[34] N. Schwierz, I. Wiedenhover, and A. Volya, arXiv:0709.3525.

[35] J. Dudek, B. Szpak, M.-G. Porquet, H. Molique, K. Rybak, and B. Fornal, J. Phys. G37, 064031 (2010).

[36] J. Dudek, B. Szpak, M.-G. Porquet, and B. Fornal (unpublished).

[37] M.-G. Porquet (private communication).

[38] J. Dobaczewski, W. Nazarewicz, and P.-G. Reinhard, J. Phys. G41, 074001 (2014).

[39] M. Kortelainen, J. Dobaczewski, K. Mizuyama, and J. Toivanen, Phys. Rev. C 77, 064307 (2008).

[40] Y. M. Engel, D. M. Brink, K. Goeke, S. J. Krieger, and D. Vautherin, Nucl. Phys. A249, 215 (1975).

[41] E. Perlińska, S. G. Rohoziński, J. Dobaczewski, and W. Nazarewicz, Phys. Rev. C 69, 014316 (2004).

[42] A. Björck, Numerical Methods for Least Squares Problems (Society for Industrial and Applied Mathematics, Philadelphia, PA, 1996).

[43] J. Toivanen, J. Dobaczewski, M. Kortelainen, and K. Mizuyama, Phys. Rev. C 78, 034306 (2008).

[44] K. Mizuyama, G. Colò, and E. Vigezzi, Phys. Rev. C 86, 034318 (2012). 